\title{
Simplified Modeling of the Evolution of Skills in a Spatially Resolved Environment
}

\author{
Rudolf M. Füchslin ${ }^{1,2}$, Johannes J. Schneider ${ }^{1}$, Thomas Ott ${ }^{3}$ and Richard Walker ${ }^{1}$ \\ ${ }^{1}$ Zurich University of Applied Sciences, School of Engineering, Winterthur, Switzerland \\ ${ }^{2}$ European Centre for Living Technology, Venice, Italy \\ ${ }^{3}$ Zurich University of Applied Sciences, School of Life Sciences, Wädenswil, Switzerland \\ rudolf.fuechslin@zhaw.ch
}

\begin{abstract}
We present a model for the spread, transmission and competition of skills with an emphasis on the role of spatial mobility of individuals. From a methodological point of view, we seek mathematical and computational simplicity in the sense of a minimal model. This minimalism lets us use a infinite dimensional simplex space and not a Euclidean space as underlying structure. Such a simplex captures the essentials of spatial heterogeneity without the mathematical difficulties of neighborhood structures.

In the presented model, individuals may have no skill or either skill A or B. Individuals are born unskilled and may acquire skills by learning from a skilled individual. Skill $\mathrm{A}$ results in a small reproductive advantage and is easy to transmit (teaching happens at high rate), whereas skill $\mathrm{B}$ is harder to teach but results in a high benefit. The model exhibits a rich behavior; after an initial transient, the system settles to a fix point (constant distribution of skills), whereby the distribution of skills depends on a mobility parameter $\mathrm{m}$. We observe different regimes, and as the main result, we conclude that for some settings of the system parameters, the spread of the (harder to learn but more beneficial) skill $\mathrm{B}$ is only possible within a specific range of the mobility parameter.

From a technical point of view, this paper presents the application of the PRESS-method (probability reduced evolution of spatially resolved species) that enables the study of spatial effects in a very efficient manner. We analyze the consequences of spatial organization and argue that we can study aspects of social dynamics in an infinite dimensional simplex space. In spite of this maybe daunting name, the dynamics on such a structure is comparably easy to implement.

The model we present is far from reflecting all the details of human interaction. On the contrary, we deliberately tailored the model to be as simple as possible from a mathematical point of view (but still reflecting central properties of spatial organization). This approach is guided by physics, where seemingly simple models which obviously don't reflect the true physical behavior of a system (such as the Ising model) are nevertheless suited to reveal fundamental aspects and limiting cases of the real world.
\end{abstract}

\section{Modeling Social Dynamics: Methodological Considerations}

Studying social dynamics has to combine two core elements:

1. The interaction of individuals, once they meet.

2. The conditions and mechanisms that they meet.
Thereby, "to meet" implies the existence of an (implicit or explicit) binary function that determines whether two individuals are in contact. The notion of contact invokes aspects of space. At least, it requires a concept of "location" that enables to distinguish for a pair of individuals whether they are at the same location or not.

There are two main approaches for modeling and simulating social and societal dynamics: Agent-based [1-3] and densitiybased [4-6] simulations, the latter relying on systems of ordinary differential equations (ODE).

The agent-based simulation: The modeling consists in the precise determination of the interaction of individuals and the way, how and when this interaction takes place. The simulation platform translates these descriptions of interactions into actual encounters between individuals happening at rates resulting from the way how the individuals move. Thereby, "movements" have to be understood in a very general sense; the underlying space in which these movements take place can be an abstract one, for example a network.

Agent-based modeling exhibits a number of advantages.

a. Simulating spatial effects is easy; agents just need a position attribute that represents their location in some form of spatial structure. Social interaction can easily be a function of these attributes.

b. It is comparably easy to implement a combinatorial variety of interactions; an agent can be equipped with a table of attributes and interaction between two individuals can be determined as a function of the entries in the respective two attribute tables.

c. From a technical perspective, it is no problem to introduce novel types of individuals (novel combinations of attributes) during a running simulation.

d. Agent-based simulations exhibit fluctuations; this can be of advantage if one wants to include fluctuations into the simulation.

However, there are a number of challenges:

a. Agent-based simulations scale quadratically (or with some other exponent larger than 1) with the number of individuals involved, e.g., if each individual gets the chance to interact with any other individual [7-9].

b. Agent-based simulations exhibit fluctuations; this can be a disadvantage if one is interested in average values. Then, large numbers of agents are required, resulting in an according amount of computing time. 
An alternative are approaches based on systems of ordinary differential equations: in such studies, one treats social dynamics in a way similar to chemical reaction kinetics; to be precise: chemical reaction kinetics in a well-stirred pot, means a homogeneous reaction environment.

Advantages of the ODE approach are:

a. They work (at least implicitly) with continuous densities. No fluctuations due to discretization effects occur. In other words: One always works in the limit of infinitely many individuals.

b. In rare cases (usually for the limits of some parameter values), analytical solutions can be obtained. To be precise, this happens very rarely for the study of dynamics but more often for the study of fixed points.

Some disadvantages balance these benefits:

a. In a well-stirred reaction environment, there is no notion of space. As soon as the probability for the contact of two individuals requires a more involved conceptual framework than that of a collision frequency in chemical kinetics, ODE approaches become quite often somewhat contrived.

b. ODE approaches exhibit unfavorable scaling behavior if one considers bigger numbers of different types of individuals.

c. It is technically very hard to change the number of types during a running simulation (one has to construct a new set of ODE).

In what follows, we describe an approach that is based on systems of ordinary differential equations and nevertheless captures essential aspects of spatial structuring in a natural way, the so-called probability reduced evolution of spatially resolved species approach (PRESS). The method is based on an idea of John S. McCaskill and has been described in a series of publications with emphasis on evolutionary dynamics in molecular biology [10-12].

\section{The PRESS Approach}

The PRESS approach assumes a network of locations. Interactions happen in the locations. The locations are connected and migration may take place from one location to another one. The number of sites in a location is finite and fixed and the same for all locations. Also limited is the number of different types of individuals. In consequence, a location can only attain a finite number of states. The PRESS approach assumes the network of locations to be a simplex (each location has the same number of sites and is connected to all the other locations). This inherent symmetry implies that the probability for a location to be in a specific state is well-defined and the same for all locations. The goal of the PRESS approach is to compute the dynamics of these probabilities. From a mathematical perspective (since all the locations are fundamentally equal and each location is connected to all the other locations), migration can be expressed in terms of a mean field approach (as will be detailed in what follows).

Why using a simplex? The geometrical concept "space" carries a surprisingly rich variety of mathematical structure; most fundamentally, a notion of space means that one can distinguish between here and there. In addition, spaces such as our familiar three-dimensional Euclidean space bears structure that allows to quantify the "theres" (means "non-heres") by a notion of distance. The "theres" can be grouped in locations that are far or nearer to the "here". Furthermore, the notion of space, especially if one considers nontrivial spaces, always contains connections, that means paths from a "here" to a "there". These connections are trivial in the case of Euclidean spaces, but become more interesting in the spaces studied in higher mathematics as well as in those occurring in everyday life (e.g. the road system that connects different cities makes it a nontrivial decision of how to get from Lausanne to Zurich.)

We mentioned above that simulations based on systems of ordinary differential equations most often relate to models in which we make the assumption of a "well - stirred reaction environment". This means nothing else then the complete absence of spatial structure. Despite its daunting name, an infinite-dimensional simplex is probably the smallest possible step from a completely homogeneous reaction environment towards a model that captures at least the most fundamental aspects of space. For our purposes, a simplex is nothing more than a set of $n$ discrete locations which are all mutually connected in an identical manner (s. $n=4,5,6$ - simplices in Fig. 1.) An infinite dimensional simplex is then the limiting case of large numbers of locations [13]. The question "Why a simplex?" is answered by noting that it is the simplest structure representing some sort of spatial heterogeneity but without all the computational challenges resulting from heterogeneous neighborhood structures (two locations may be in different states, but on simplex, they all have the same neighborhood, namely all the other locations).

In a social context, such a simplex can be interpreted as a densely packed village that consists of identical, small huts. These huts are the places where the interaction between individuals take place, see Fig. 2. Since we model only unary and binary interactions, locations which can host up to maximally two reaction partners constitute a minimal reaction environment. The goal of the model presented is then to compute the probability for each possible occupation state.

In our model, we make the following assumptions:

a. A hut can host up to two individuals.

b. Individuals only interact inside huts.

c. There may be different types of individuals; the interaction between two individuals is a function of their respective types.

d. The individuals commute freely between huts. If an individual leaves a hut, it chooses a different hut to enter at random.

e. The time needed for the passage between huts is negligible.
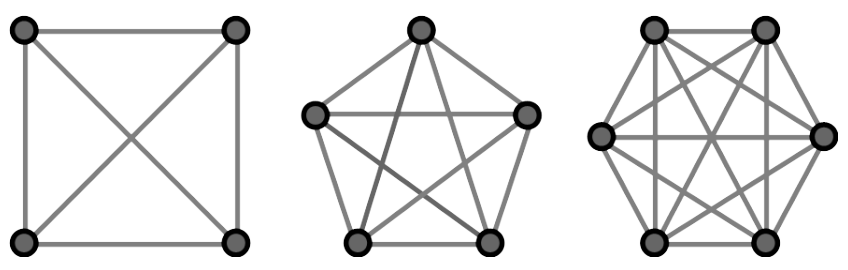

Fig. 1: Simplices for $n=4,5,6$.

Besides the fact that in our model the village is highly symmetric, we introduce a further idealization. We model the village in the limit of a very large number of huts. The rational 
for his approach is given by the fact that we are interested in the number of huts with a specific occupation, i.e. a specific combination of individuals (say, the number of huts occupied by only one individual of the blue type or the number of huts hosting a blue and a red individual). Knowing the numbers of huts for each possible occupation, we can easily compute the total number of individuals of a given type.

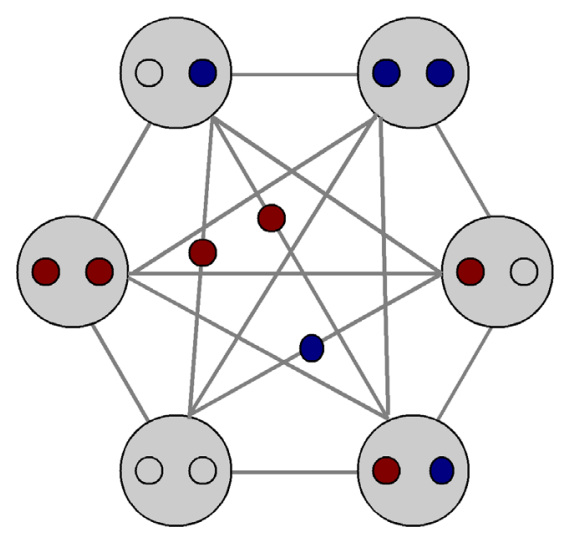

Fig. 2: A simplex built up from locations, which can host up to two individuals.

For a finite number $n$ of huts, the number $n_{X}$ of huts with a specific occupation $X$ is a discrete value. Moreover, if the interaction between individuals and the passage between huts is driven by random events, $n_{X}$ will be subject to fluctuations. Instead of $n_{X}$, we will compute the probability $p_{X}$ for a hut to have an occupation $X$; it simply holds

$$
p_{X}=\frac{n_{X}}{n}
$$

In the limit of large $n, p_{X}$ will become a continuous variable. In addition, $p_{X}$ will not exhibit fluctuations (without a detailed proof and referring to the central limit theorem, we assume the fluctuations $\Delta n_{X}$ of $n_{X}$ to scale in a sub - linear way with $n_{X}$. The fraction $\Delta n_{X} / n_{X}$ will then vanish for large $n$ ).

In what follows, we will set up the dynamics of the probabilities for the various possible occupations of the huts.

This approach, which will be exemplified in the next section, has already been introduced in the context of chemical reaction kinetics [10-12]. The authors called it the PRESS - approach, for Probability Reduced Evolution of $\underline{\text { Spatially resolved }}$

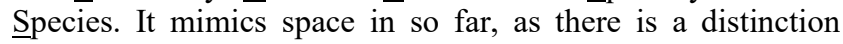
between different locations and as there is a parameter, namely a rate constant $m$ for the passage from one hut to the other. The mobility $m$ can be understood as the analogue to the diffusion constant in Euclidean space. It is also a parameter that can be interpreted in terms familiar to the social sciences as a type of migration frequency.
Obviously, our modeling framework represents a highly idealized situation. Whether the metaphor of a hut is appropriate may be discussed. Certainly, the restriction to a maximal occupation number of two is "unrealistic" in the sense that real huts can easily be larger. Understanding the huts as interaction sites enables a more abstract view. A restriction to size two can be justified in various ways. One way is the already mentioned perspective on the maximal number of partners in the modeled social interactions. As will be discussed below, this is two in our case. Another way of looking at this restriction is coarse graining. The interaction locations can be in a number of different states and we compute their respective probabilities based on a set of interactions. Assuming locations with two sites spans the minimal set of states necessary for representing the transitions we want to model. Further idealizations refer to the various symmetries invoked in our model. In a real village, huts will not be identical, interactions between individuals will not only take place inside huts, the huts will belong to somebody and not be chosen randomly and the distance between the huts will play a role for the probability of a transfer from hut A to hut B.

Furthermore, a simplex needs not necessarily to be understood as a village consisting of huts. One may understand the individual interaction sites as caricatures of villages themselves. The whole simplex then is a collection of interacting small villages. The occupation of a village may represent its internal state. Transport between interaction sites can be understood as information flows between isolated, but weakly interacting groups of human beings.

\section{Modeling the Competition of Skills}

Our goal is to study the question under which conditions (means for which parameter values) different skills are spread and maintained in populations. Thereby, a skill is a property of an individual that a) gives the individual some benefit and b) has to be transferred from a skilled to an unskilled individual by some form of teaching process. We will study a population in which individuals can learn one of two skills. These two skills differ first in the benefit they provide to dose individual having the particular skill and second in the effort (time) it takes to teach the skill to an unskilled individual U. In the model, there is an easy-to-learn skill A that yields only limited benefit and a skill B that is hard to learn but results in a higher benefit. The question we want to address is the following: What is the dependency of the average number of individuals with skill A, $B$ or $U$ (skill $U$ means unskilled) one encounters in the randomly chosen hut as a function of the mobility parameter $m$ ? This question arose from broader investigations about cultural evolution performed by one of the authors, Richard Walker. As it turns out, the dynamics of the model delivers a surprisingly faceted answer. 


\section{The Model}

\section{The Possible Occupations}

We study huts that can host maximally two individuals. Larger huts are straightforward to implement, but we want to keep the mathematical formalism as simple as possible. The symbol "V" stands for void, "U" for unskilled, "A" for individual with skill $\mathrm{A}$ and " $\mathrm{B}$ " represents an individual with skill $\mathrm{B}$. The occupation or state of a hut is described by an index consisting of three non - negative integers $U A B$, such that $U+A+B \leq 2$. The total number $T$ of individuals in a state $U A B$ is given by $T=U+A+B$ and the according number of voids by $V=2-U-A-B$. The index set $I_{1}=\{000,100,010,001,200,110,101,020,011,002\}$ contains all allowed indices for huts of size two.

Sometimes, an alternative indexation is useful, referring to the two available vacancies in a hut. We define an index set $I_{2}=\{V V, V U, V A, V B, U U, U A, U B, A A, A B, B B\}$. In this notation, a potential problem becomes immediately apparent, namely the occurrence of symmetric states. In this work, we do not distinguish between occupations $\mathrm{AB}$ and $\mathrm{BA}$.

For each occupation $U A B$, there is a time-dependent probability $p_{U A B}(t)$. We formulate a Master equation

$$
\frac{d \vec{P}}{d t}=M(\vec{P}(t)) \vec{P}, \quad \vec{P}=\left(\begin{array}{c}
p_{000}(t) \\
\vdots \\
p_{002}(t)
\end{array}\right)
$$

in order to determine the time development of the occupations. Thereby, $M(\vec{P}(t))$ is a matrix that depends on $\vec{P}(t)$ (and therefore implicitly on time) and represents the different interactions transforming one state into another. In what follows, we will discuss these interactions and the present their contribution to $M(\vec{P}(t))$.

\section{Interactions and Processes}

In our model, four types of processes can occur:

a. Individuals may give birth to children. Children are always unskilled.

b. An individual with skill $\mathrm{X}$ can teach an unskilled individual.

c. Individuals can die.

d. Individuals can leave a hut and enter another one.

In what follows, we provide the rate equations for the probabilities $p_{U A B}(t)$ for each of these processes. We will formulate template formulas. In these template formulas, we assume that $p_{U A B}(t) \equiv 0$ if the index $U A B$ does not satisfy the requirement that all the integers of which the index is composed are bigger or equal to zero or that the sum $U+A+B$ is bigger than two. To facilitate the notation further, we introduce the function $\varepsilon$ :

$$
\varepsilon(U, A, B)= \begin{cases}1, & U, A, B \geq 0, U+A+B \leq 2 \\ 0, & \text { otherwise }\end{cases}
$$

\section{Birth}

The birth rate of individuals with skill $\mathrm{X}$ is given by $\beta_{X}$, $X \in\{U, A, B\}$ (we don't model the details of sexual reproduction). Different skills may lead to different birthrates, which in turn constitute evolutionary advantages or disadvantages.

The probability $p_{U A B}(t)$ will change by births according to the following template formula:

$$
\begin{aligned}
\left.\frac{d p_{U A B}}{d t}\right|_{b i r t h} & =(U-1) \beta_{U} p_{(U-1) A B} \\
& +A \beta_{A} p_{(U-1) A B}+B \beta_{B} p_{(U-1) A B} \\
& -\sum_{X \in\{U, A, B\}} X \varepsilon(U+1, A, B) \beta_{X} p_{U A B}
\end{aligned}
$$

Let us analyze this formula. The term $p_{U A B}$ gives the probability for the state with $U$ unskilled inhabitants, $A$ inhabitants with skill $\mathrm{A}$ and $B$ inhabitants with skill $\mathrm{B}$. The first term on the right hand side, $(U-1) \beta_{U} p_{(U-1) A B}$ models the birth of an unskilled individual from an unskilled parent with a birth rate $\beta_{U}$. Because birth increments the number of unskilled individuals by one, the original state is $p_{(U-1) A B}$. The factor $(U-1)$ takes into account the possibility of many potential parents (which is not important if one restricts the maximal occupation number to two, but the template can be applied for larger huts, too.) The second term $A \beta_{A} p_{(U-1) A B}$ represents the birth of an unskilled inhabitant by a parent with skill A, and accordingly for the third term and skill B. The last term models the decrease of the probability $p_{U A B}$ caused by birth processes (Note that in our model, we could replace $\varepsilon(U+1, A, B)$ by $(2-U-A-B)$, but the variant we use is also valid, if we run the model with larger huts.) A remark: the fact that the number of vacancies in a hut is restricted imposes an implicit resource that limits growth.

\section{Death}

The template formula for the death of individuals is structurally identical to the one of birth. It reads:

$$
\begin{aligned}
\left.\frac{d p_{U A B}}{d t}\right|_{\text {death }} & =(U+1) \delta_{U} p_{(U+1) A B}+(A+1) \delta_{A} p_{U(A+1) B} \\
& +(B+1) \delta_{B} p_{U A(B+1)}-\sum_{X \in\{U, A, B\}} X \delta_{X} p_{U A B}
\end{aligned}
$$


The death rates, which may be different for differently skilled individuals, are given by $\delta_{X}$.

\section{Teaching}

We assume that if an unskilled individual resides together with an individual with skill $\mathrm{X}$ in a hut, a teaching process may occur. The according template formula reads:

$$
\begin{aligned}
\left.\frac{d p_{U A B}}{d t}\right|_{\text {teach }} & =(U+1)(A-1) \tau_{A} p_{(U+1)(A-1) B} \\
& +(U+1)(B-1) \tau_{B} p_{(U+1) \mathrm{A}(B-1)} \\
& -U A \tau_{A} p_{U A B}-U B \tau_{B} p_{U A B}
\end{aligned}
$$

The first line represents the transformation of an unskilled individual into one with skill A (the unskilled individual is taught by an individual with skill A). This transformation, the "teaching rate", is given by $\tau_{A}$. Accordingly, the second line models the teaching of skill $\mathrm{B}$ with teaching rate $\tau_{B}$. Finally, the last line represents transformations out of state $U A B$.

Remark: this template is a bit more complicated than it needs to be, at least as long as we restrict the number of vacancies in a hut to two. However, the formula as it stands can is applicable for larger huts.

\section{Diffusion}

Modeling diffusion is conceptually a little bit more difficult. First, we have to note that we assume an individual leaving a hut and entering immediately into another one. One can think of an individual choosing a target hut at random and make a passage to this hut as long as this hut is not already full (because we work on an infinite dimensional simplex, the case of leaving a hut and entering it again does not require specific care). The an individual with skill $X$ fills a vacancy in a given hut is then proportional to the average number of individuals with that skill in all the other huts. As a consequence, the chance of an individual with skill $\mathrm{X}$ in a given hut to leave this hut is proportional to the average number of vacancies in all the other huts.

These averages are easy to compute; the advantage of an infinite dimensional simplex as underlying space is that the occupation probabilities in all the hearts are identical. Therefore, we have:

$$
\begin{aligned}
\bar{U} & =\sum_{i \in I_{1}} u(i) p_{i} \\
\bar{A} & =\sum_{i \in I_{1}} a(i) p_{i} \\
\bar{B} & =\sum_{i \in I_{1}} b(i) p_{i} \\
\bar{V} & =\sum_{i \in I_{1}} v(i) p_{i}
\end{aligned}
$$

Thereby, $i$ is an index of the form $U A B$ and the functions $u, a, b$ are defined as $u(i=U A B)=U, a(i=U A B)=A$, $b(i=U A B)=B$ and $v(i=U A B)=2-U-A-B$.

The template formula for in - diffusion (state transitions that increase the number of inhabitants) reads:

$$
\begin{aligned}
\left.\frac{d p_{U A B}}{d t}\right|_{i n-d i f f}= & (2-(U-1)-A-B) \bar{U} m p_{(U-1) A B} \\
& +(2-U-(A-1)-B) \bar{A} m p_{U(A-1) B} \\
& +(2-U-A-(B-1)) \bar{B} m p_{U A(B-1)} \\
& -\sum_{X \in\{U, A, B\}}(2-U-A-B) \bar{X} m p_{U A B}
\end{aligned}
$$

Thereby, the parameter $m$ models the mobility and the last line takes into account that the influx of an inhabitant into a state $U A B$ reduces $p_{U A B}$.

Accordingly, for out-diffusion (state transitions that decrease the number of inhabitants), we have:

$$
\begin{aligned}
\left.\frac{d p_{U A B}}{d t}\right|_{\text {out-diff }}= & (U+1) \bar{V} m p_{(U+1) A B}+(A+1) \bar{V} m p_{U(A+1) B} \\
& +(B+1) \bar{V} m p_{U A(B+1)}-\sum_{X \in\{U, A, B\}} X \bar{V} m p_{U A B}
\end{aligned}
$$

The complete Master equation is given by:

$$
\begin{aligned}
\frac{d p_{U A B}}{d t} & =\left.\frac{d p_{U A B}}{d t}\right|_{\text {birth }}+\left.\frac{d p_{U A B}}{d t}\right|_{\text {death }}+\left.\frac{d p_{U A B}}{d t}\right|_{\text {teach }} \\
& +\left.\frac{d p_{U A B}}{d t}\right|_{\text {in-diff }}+\left.\frac{d p_{U A B}}{d t}\right|_{\text {out-diff }}
\end{aligned}
$$

\section{Results}

We solved the Master equation Eq. (10) by standard numerical procedures. As it turned out, the solutions settled down to a fixed point after a sufficiently long time development. Of course, many parameter values lead to rather uninteresting behavior. However, some showed a rather rich behavior. In what follows, we analyze the system for the following set of parameters: 


$$
\begin{aligned}
& \beta_{U}=1 / 10 \\
& \beta_{A}=1 / 5 \\
& \beta_{B}=1 / 2.5 \\
& \delta_{U}=\delta_{A}=\delta_{B}=1 / 80 \\
& \tau_{A}=1 / 5 \\
& \tau_{B}=1 / 10
\end{aligned}
$$

If one takes the unit of time as one year, one sees that the average life span of an individual is 80 yrs. Learning a skill takes 5 years (skill A) or ten years (skill B). The birth rates look much larger as they really are; take into account that the number of births is limited (space is a limited resource).

For this choice of parameters, we observe a rich variety of possible fix points. In Fig. 3, we distinguish seven different patterns of behavior with respect to the mobility parameter $m$. Shown are the values the averages of skills $U, A, B$ attained after $10^{6}$ time units. The initial conditions are set to $\bar{V}(0)=1, \bar{U}(0)=0.05, \bar{A}(0)=\bar{B}(0)=0.225$. The system turned out to be insusceptible towards the initial conditions, provided that there is at least some density of the skills A and B.

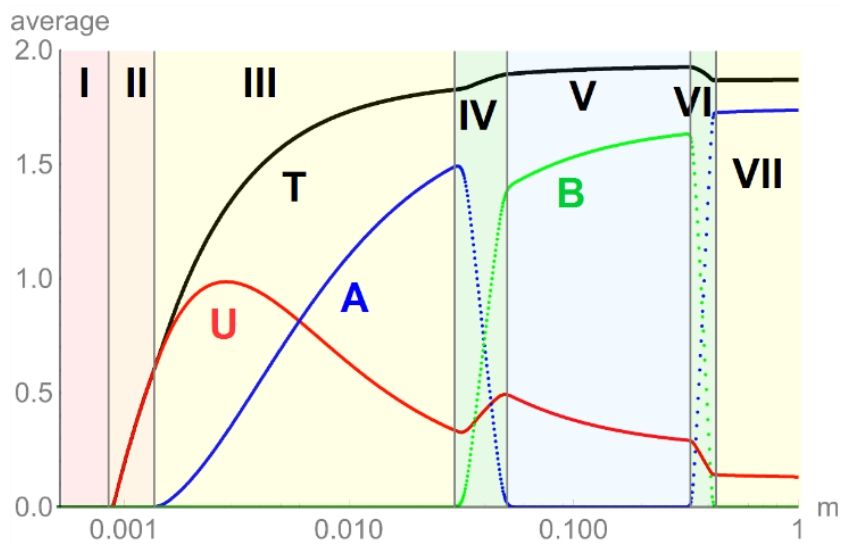

Fig. 3: Average number of skilled individuals per location of interaction as a function of the migration parameter $m$.

In Fig. 3, the y-axis reflects the expected average density of a given skill as a function of the mobility parameter $\mathrm{m}$. $U$ represents the density of unskilled individuals, A and B those of individuals with the respective skill. T stands for the total of individuals. At very low $\mathrm{m}$ (region I), the population can't survive. For low m (region II), no skill can be maintained. For higher values of $\mathrm{m}$, we observe either that one skill dominates the other or co - existence of skills. As a main result, we observe that a population can maintain a complex skill B only in a window of the mobility parameter. In order to uphold a complex skill, a certain mobility is necessary, but too much mobility favors simpler skills that are easier to transmit.

\section{Discussion}

The outcome of our simulations shows that maintaining skills in a population may depend on migration rates in a rather subtle manner. However, we are very reluctant giving these simulations (or the parameter values at which changes in behavior of the system occur) a lot of direct relevance. And of course, we are far from modeling the real processes in prehistoric societies. But physics teaches us that seemingly simple models which obviously don't reflect the true physical behavior of a system (such as the Ising model) are nevertheless suited to reveal fundamental aspects and limiting cases of the real world. In that sense, we are convinced that a PRESS model based approach to social dynamics can help us understand and guide our search for interactions, which lead to interesting system behavior.

An example for such a support is a question that results from the observations in Fig. 3. Basically, Fig. 3 states a complex dependence of the system behavior on the migration rate. But in the presented model, a migration process only takes place if a randomly chosen hut has at least one empty site. With other words: migration is limited by the number of free sites and there is no spread of information different from the migration of individuals. However, one can easily imagine a situation in which the flow of information is much faster than migration of individuals. This could e.g. lead to a migration pattern where potential students (unskilled individuals) know in advance which hut harbors empty sites. Technically, this means that migration stays maximal until the system is full.

Investigations based on the PRESS model should (and will) be complemented by agent based simulations. In an agent-based simulation, it is very easy to study the effect of a fast flow of information.

\section{Acknowledgements}

This work has been partially supported by the European Horizon 2020 project ACDC - Artificial Cells with Distributed Cores to Decipher Protein Function ", grant agreement No 824060". Relevant parts of the mathematical framework developed in ACDC have been used in this work.

\section{References}

1. S. Moss de Oliveira, P. M. C. de Oliveira, and D. Stauffer, Evolution, Money, War, and Computers, Teubner, Stuttgart, Leipzig, 1999, ISBN 9783-519-00279-6.

2. J. J. Schneider and C. Hirtreiter, The Impact of Election Results on the Member Numbers oft he Large Parties in Bavaria and Germany, Int. J. Mod. Phys. C Vol. 16, 1165-1215, 2005.

3. S. Kirkpatrick and J. J. Schneider, How Smart Does an Agent Need to Be?, Int. J. Mod. Phys. C Vol. 16, 139-155, 2005.

4. R. Hesan, A. Ghorbani, and V. Dignum, Using Difference Equation to Model Discrete-time Behavior in System Dynamics Modeling, $32^{\text {nd }}$ International Conference of the System Dynamics Society, Delft, The Netherlands, 2014 , https://pdfs.semanticscholar.org/0832/553cfa88aa069fb3335cb040aac779 97e1f0.pdf. 
5. C. M. Brown, Differential Equations - A Modeling Approach, Series Quantitative Applications in the Social Sciences Vol. 150, Sage Publishing, Los Angeles, 2007, ISBN 9781412941082.

6. H. Van Dyke Parunak, R. Savit, and R. L. Riolo, Agent-Based Modeling vs. Equation-Based Modeling: A Case Study and Users' Guide, International Workshop on Multi-Agent Systems and Agent-Based Simulation, MABS, Lecture Notes in Artificial Intelligence Vol. 1534, 10 25, Springer, 1998.

7. A. Ebersbach, J. Schneider, I. Morgenstern, and R. Hammwöhner, The Influence of Trucks on Traffic Flow - An Investigation on the NagelSchreckenberg-model, Int. J. Mod. Phys. C Vol. 11, 837-842, 2000.

8. J. J. Schneider and C. Hirtreiter, Scaling Laws fort he Lifetimes of Governments in the Sznajd Democracy, Int. J. Mod. Phys. C Vol. 16, 157$165,2005$.

9. J. J. Schneider and C. Hirtreiter, The Democracy-OchlocracyDictatorship Transition in the Sznajd Model and in the Ising Model, Physica A Vol 353, 539-554, 2005.

10. J. S. McCaskill, R. M. Füchslin, and S. Altmeyer, The stochastic evolution of catalysts in spatially resolved molecular systems, Biol. Chem. Vol. 382, No. 9, 1343-1363, 2001.

11. R. M. Füchslin, S. Altmeyer, and J. S. McCaskill, Evolutionary stabilization of generous replicases by complex formation, Europ. Phys J. B Vol. 1, 103-110, 2004.

12. S. Altmeyer, R. M. Füchslin, and J. S. McCaskill, Folding stabilizes the evolution of catalysts, Artificial Life Vol. 10, 23-38, 2004.

13. https://mathworld.wolfram.com/CompleteGraph.html 